\title{
A first order semantic approach to adjectival inference
}

\author{
Marilisa Amoia \\ INRIA/Université de Nancy 1 \& \\ University of the Saarland \\ Saarbrücken, Germany \\ amoia@coli.uni-saarland.de
}

\author{
Claire gardent \\ CNRS/Loria \\ Campus Scientifique BP 239 \\ 54506 Vandoeuvre-les-Nancy, France \\ claire.gardenteloria.fr
}

\begin{abstract}
As shown in the formal semantics literature, adjectives can display very different inferential patterns depending on whether they are intersective, privative, subsective or plain non-subsective. Moreover, many of these classes are often described using second order constructs. In this paper, we adopt Hobbs's ontologically promiscuous approach and present a first order treatment of adjective semantics which opens the way for a sophisticated treatment of adjectival inference. The approach was implemented and tested using first order automated reasoners.
\end{abstract}

\section{Introduction}

As has often been observed, not all of natural language meaning can be represented by first order logic. There are expressions such as, most, former, I didn't whose meaning intuitively involve higherorder constructs.

Nevertheless, as (Hobbs, 1985) and others have argued, semantic representations for natural language need not be higher-order in that ontological promiscuity can solve the problem. That is, by reifying all objects that can be predicated of, it is possible to retain a semantic representation scheme for NL that is first-order.

This observation is crucial for computational applications for two reasons. First, logics that goes beyond first order are highly undecidable. Second and more importantly, there is no off the shelf higher or- der automated reasoners that could be put to use to reason about the meaning of higher-order formulae.

In this paper, we present a semantics for adjectives that adopts an ontologically promiscuous approach and thereby supports first order inference for all types of adjectives including extensional ones.

Indeed, traditional semantic classifications of adjectives such as (Chierchia and Connell-Ginet, 1990; Kamp, 1975; Kamp and Partee, 1995) subdivide adjectives into two classes namely extensional vs. intensional adjectives, the latter grouping together adjectives which intuitively denote functions from properties to properties, i.e. second order objects.

We present a compositional semantics for adjectives which both (i) defines a first order representation and (ii) integrates interactions with other sources of linguistic information such as lexical semantics and morpho-derivational relations. We then show that the proposed semantics correctly predicts the inferential patterns observed to hold of the various adjective subclasses identified in the literature (Chierchia and Connell-Ginet, 1990; Kamp, 1975; Kamp and Partee, 1995; Amoia and Gardent, 2006).

This paper is structured as follows. We start by presenting a classification of adjectives which is motivated by the different inferential patterns observed. We then propose a compositional semantics for each class and show that it correctly predicts their inferential behaviour. We conclude with a brief discussion of related work and pointers for further research.

\section{Inferential patterns and adjective classes}

In the literature (Chierchia and Connell-Ginet, 1990; Kamp, 1975; Kamp and Partee, 1995; Amoia and 
Gardent, 2006), adjectives are usually divided into four main classes namely, intersective, subsective, privative and plain non subsective depending on whether or not the $[\text { Adj } \mathrm{N}]_{A P}$ phrase entails the properties expressed by the noun and/or the adjective. More specifically, each of the four classes is characterised as follows.

Intersective adjectives. This class includes common categorical (e.g., red, rectangular, French) and tautological (e.g., real, present) adjectives. It is characterised by the inferential patterns:

$$
\begin{aligned}
& {\left[\begin{array}{ll}
A & N
\end{array}\right]=N} \\
& {[A]=A}
\end{aligned}
$$

For instance, saying that there is a red table implies both that there is something red and that there is a table.

Subsective adjectives form an ontologically heterogeneous class including for instance denominal (e.g., gastronomical) and measure (e.g. big) adjectives. They are characterised by the fact that the [Adj $\mathrm{N}]_{A P}$ phrase does not entail the Adj property:

$$
\begin{aligned}
& {\left[\begin{array}{ll}
A & N
\end{array}\right]=N} \\
& {[A N] \quad \forall A}
\end{aligned}
$$

For instance, a big mouse is a mouse but is not big. Instead it is "big for a mouse". In other words, 'bigness' cannot be directly inferred as, e.g. a big mouse and a big elephant are big in very different ways.

Privative adjectives denote adjectives such that the $[\text { Adj } \mathrm{N}]_{A P}$ phrase entails the negation of the $\mathrm{N}$ property:

$$
[A N] \quad \models \neg N
$$

For instance, the former king is not the king and $a$ fake weapon is not a weapon.

Plain non-subsective adjectives are adjectives which preclude any inference wrt to the $\mathrm{N}$ property:

$$
\begin{aligned}
& {[A N] \quad=(N \vee \neg N)} \\
& {[A N] \quad \neq A}
\end{aligned}
$$

Thus, if Peter is an alleged murderer, it is impossible to know whether or not he is a murderer.
Now, the class of intensional adjectives groups together adjectives with a syntactic and semantic idiosyncratic behaviour. Syntactically, intensional adjectives are not gradable (e.g. cannot be modified by very) and most of them can only be used attributively (He is a former president but not The president is former). Semantically, they are usually taken to denote second order properties, i.e. functions of the type $\langle\langle e, t\rangle,\langle e, t\rangle\rangle$.

Intensional adjectives include denominal (or relational) adjectives (e.g polar bear, atomic scientist), manner (or adverbial) adjectives (e.g. a poor liar, $a$ fast car), emotive (e.g. a poor man) and modals, i.e. all adjectives which are related to adverbs, quantifiers or determiners (e.g. a feeble excuse, the specific reason, a fake nose, etc.).

\section{Assigning FOL Representation to Intensional adjectives}

We now show how adjectives can be assigned an appropriate first order logic representation which appropriately reflects their inferential behaviour.

Following Hobbs, we adopt a promiscuous ontology and assume that for every predication that can be made in natural language, there corresponds an "eventuality". As Hobbs has argued, this allows for higher order predications to remain first order in that they become predications over (first order) eventualities.

Thus, in the domain there are entities which are either eventualities or individuals and relations between individuals. Moreover like Hobbs, we assume a model to describe a platonic universe containing everything that can be spoken about whether or not these things exist in the real world. To express existence in the real world, a special predicate (Exists) is introduced.

We use the following notation:

- $\mathrm{e}_{i}$, for eventuality variables,

- $\mathrm{x}_{i}$, for individuals,

- $\mathrm{P}_{i}$, for properties of individuals.

And the following types:

- e will denote the type of individuals,

- ev the type of eventualities and 
- $t$ a truth value.

\subsection{The intuition}

As shown in section 2, the semantics of $[\operatorname{Adj} \mathrm{N}]_{A P}$ phrases has very different inferential properties depending on the type of the adjective Adj. The differences stem from three main points.

The number of individuals introduced by the

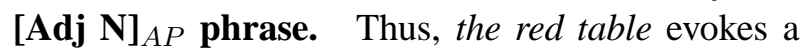
single individual $x$ which is both red and a table whilst the gastronomical book refers to a book $x$ which is about the gastronomy concept $y$. More generally, the variables predicated of by the noun and by the adjective can refer either to the same or to two distinct individual(s).

The properties licensed by the adjective and the noun to contribute to the meaning of the [Adj $\mathbf{N}]_{A P}$ phrase. Depending on the adjective type, the properties denoted by Adj and $\mathrm{N}$ will contribute either directly or indirectly to the meaning of the [Adj $\mathrm{N}]_{A P}$ phrase. Thus in an intersective [Adj $\mathrm{N}]_{A P}$ phrase, the meaning contributed by Adj and $\mathrm{N}$ are simply the properties they denote. By contrast, the privative fake forces the negation of the $\mathrm{N}$ property to be part of the Adj $\mathrm{N}$ meaning whilst the subsective gastronomical induces a relation to the morphoderivationally related noun concept (about gastronomy) to be included in the the Adj $\mathrm{N}$ meaning. More generally, the properties that compose the meaning of the Adj $\mathrm{N}$ phrase can be the denotation of Adj and/or N, the negation of N, its denotation in the past or some property derived from it.

The existence in the real world of the entity denoted by the NP. In all cases the $[\operatorname{Adj} \mathrm{N}]_{A P}$ phrase denotes a set of individuals but whilst in most cases the $[\operatorname{Adj} \mathrm{N}]_{A P}$ phrase is neutral with respect to the existence in the real world of these individuals, plain non-subsective [Adj $\mathrm{N}]_{A P}$ phrases (e.g. alleged murderer) explicitly question it (an alleged murderer may or not exist in the real world).

\subsection{The semantics of nouns}

In designing a semantics for adjectives, we assume a semantics for nouns which reflect their possible interactions with the different types of adjectives

(1) a. noun: $\lambda \operatorname{Pol} \lambda e \lambda x \cdot[\operatorname{Pol}(\operatorname{table}(e)) \wedge e=x]$
As we shall shortly see, the additional lambda variable $e$ is imposed by the treatment of adjective semantics we propose and more specifically by the necessity to sometimes distinguish between the individual described by the noun and the individual described by the adjective. The variable $\mathrm{Pol}$ accounts for the polarity of the noun, i.e. whether it occurs with the negation or not.

We give here also the semantics assigned to the pronouns someone/something which will be used in the derivations throughout this paper:

(2) a. someone/something: $\lambda P \exists x \cdot P(x)$

\subsection{The semantics of the copula}

Following the proposal of Mantague, we assign a unique representation for both the uses of the copula in identity statements (e.g. John is Mary $\rightarrow$ john=mary) and in predicative assertions (e.g. John is a $\operatorname{man} \rightarrow \operatorname{man}(\mathrm{john})$ ):

(3) a. be: $\lambda K \lambda x \cdot K(\lambda y(x=y))$

In the case of predicative assertions in which the predicate is an adjective (e.g. John is brave), we adjust the type of the argument of the copula in the following way:

(4) a. be Adj: $\operatorname{be}(\operatorname{Adj}(\lambda \operatorname{Pol} \lambda e \lambda x . t r u e))$

\subsection{The semantics of adjectives}

Given such a representation for nouns, we represent adjectives using the schema given in Figure 1.

Briefly, schema 1 captures the observations made in section (3.1) as follows. First it introduces an existential quantification (in the platonic universe) over not one but two variables $\left(e_{a}\right.$ and $\left.e_{n}\right)$ - depending on how the formula is instantiated (and in particular on the value of $R_{1}$ and $R_{2}$ ) these two variables may or not denote the same object. This accounts for the first observation according to which an $[\text { Adj } \mathrm{N}]_{A P}$ phrase may refer to either one or two individuals.

Second, the meaning of the $[\operatorname{Adj} \mathrm{N}]_{A P}$ phrase is a function not of the Adj and $\mathrm{N}$ meaning but rather of properties derived from these meanings ( $A^{\prime}$ for Adj and $N$, as modified by its three arguments, for $\mathrm{N}$ ). This accounts for the second observation.

Third, the use of the exists predicate will permit distinguishing between existence in the universe of discourse and existence in the real world. 


$$
\lambda N \lambda x \exists e_{a} \exists e_{n} \cdot\left[A^{\prime}\left(e_{a}\right) \wedge R_{1}\left(x, e_{a}\right) \wedge R_{2}\left(e_{n}, e_{a}\right) \wedge N(P o l)\left(e_{n}\right)(x)\right]
$$

with $A^{\prime}$ the property licensed by the adjective, $R_{1}, R_{2}$ two arbitrary relations licensed by the adjective, $N$ the property denoted by the noun and $\mathrm{Pol}$ a polarity argument of value either $\lambda S . S$ or $\lambda S . \neg S$

Figure 1: Semantics schema for all adjectives

We now show how this general schema receives different instantiations depending on the adjectival class being considered; and how each instantiation predicts the correct inferential pattern for the four adjectival classes.

\subsubsection{Intersective adjectives}

The semantic representation of an $[\mathrm{Adj} \mathrm{N}]_{A P}$ adjectival phrase involving an intersective adjective is given in Figure 2 together with the derivation of the [Adj $\mathrm{N}]_{A P}$ phrase red table. As can be seen, in this case, the relation $R_{1}$ holding between the lambda bound variable $x$ and the entity introduced by the adjective is one of identity. Similarly, the entity $e_{n}$ introduced is equated with $x$ and the relation $R_{2}$ is $\lambda x, y$.true (i.e. there is no modifying relation between $e_{a}$ and $e_{n}$ ). Hence the $[\text { Adj } \mathrm{N}]_{A P}$ phrase licenses in effect a single entity $x$ and the resulting semantics is the traditional $\lambda x .[A(x) \wedge N(x)]$ with $A$ the semantics of the adjective and $N$ that of the noun. Assuming further that determiners have the semantics:

a/the $\quad \lambda P \lambda Q \exists x \cdot[P(\lambda S . S)(x) \wedge Q(x)]$

then the semantics of Something is a red table is

(5) $\exists x \exists e_{a} \exists e_{n} \cdot\left[\operatorname{red}\left(e_{a}\right) \wedge x=e_{a} \wedge\right.$ table $\left(e_{n}\right) \wedge e_{n}=$ $x]$

which correctly entails that there is an entity $x$ which is both red and a table i.e.,

(5) $\models \exists x .[\operatorname{red}(x)]$

(5) $\models \exists x$. $[\operatorname{table}(x)]$

something is red something is a table

\subsubsection{Subsective adjectives}

As recalled above, subsective adjectives are characterised by the fact that the $[\text { Adj } \mathrm{N}]_{A P}$ phrase entails $\mathrm{N}$ but not $\mathrm{A}$. Relatedly, the adjective phrase introduces not one but two individuals, one linked to the adjective and the other to the noun. For instance, the phrase the gastronomical book refers to a book $x$ which is about the gastronomy concept $e_{n}$.

Thus in such cases, we take the $R_{2}$ relation holding between $x$, the NP quantified variable, and $e_{a}$, the entity introduced by the adjective, to be distinct from identity, while the $R_{1}$ relation is empty.

(6) $\exists x \exists e_{a} \exists e_{n} \cdot\left[\right.$ gastronomy $\left(e_{a}\right) \wedge$ about $\left(e_{n}, e_{a}\right) \wedge$ $\left.\operatorname{book}\left(e_{n}\right) \wedge e_{n}=x\right]$

This ensures that the NP refers to two entities, one bound by the determiner and licenced by $\mathrm{N}$, the other existentially quantified and licensed by A. For instance, the sentence John read every gastronomical books is interpreted as meaning that John read all books that are about gastronomy.

More generally, this ensures that $[A N] \not \models A$ (and in fact, adjectives like gastronomical cannot be used predicatively), e.g.

$$
\begin{aligned}
& =\operatorname{something} \text { is a book } \\
& =\exists x \cdot[\operatorname{book}(x)] \\
& =\operatorname{something} \text { is about gastronomy } \\
& =\exists x \exists e_{a} \cdot\left[\text { about }\left(x, e_{a}\right) \wedge \text { gastronomy }\left(e_{a}\right)\right] \\
& \not=\operatorname{something} \text { is a book and a gastronomy } \\
& \not \exists \exists[\text { book }(x) \wedge \text { gastronomy }(x)] \\
& \not \neq \operatorname{something} \text { is gastronomical } \\
& \not \neq \exists x[\text { gastronomical }(x)]
\end{aligned}
$$

As shown in (Amoia and Gardent, 2006), subsective adjectives can be further divided into at least four classes. Because of space restrictions, we only show here how to represent two of these subclasses namely denominal (e.g. gastronomical) and measure subsective adjectives (e.g. big). In both cases, the idea is to decompose the meaning of the adjectives into a finer grained lexical meaning. Depending on the lexical meaning involved, this decomposition induces different instantiation patterns for the 


\begin{tabular}{|l|}
\hline Intersective Adjectives \\
$\lambda N \lambda x \exists e_{a} \exists e_{n} \cdot\left[A\left(e_{a}\right) \wedge x=e_{a} \wedge N(\lambda S . S)\left(e_{n}\right)(x)\right]$ \\
Red table \\
$\lambda N \lambda x \exists e_{a} \exists e_{n} \cdot\left[\operatorname{red}\left(e_{a}\right) \wedge x=e_{a} \wedge N(\lambda S . S)\left(e_{n}\right)(x)\right](\lambda \operatorname{Pol} \lambda e \lambda x .[\operatorname{Pol}(\operatorname{table}(e)) \wedge e=x])$ \\
$\left.\equiv \lambda x \exists e_{a} \exists e_{n} \cdot\left[\operatorname{red}\left(e_{a}\right) \wedge x=e_{a} \wedge \operatorname{table}\left(e_{n}\right) \wedge e_{n}=x\right]\right)$ \\
$\equiv \lambda x \cdot[$ red $(x) \wedge$ table $(x)])$
\end{tabular}

Figure 2: Semantics of Intersective Adjectives

Subsective Adjectives
$\lambda N \lambda x \exists e_{a} \exists e_{n} \cdot\left[A^{\prime}\left(e_{a}\right) \wedge R_{2}\left(e_{n}, e_{a}\right) \wedge N(\lambda S . S)\left(e_{n}\right)(x)\right]$
with $A^{\prime}$ an arbitrary complex relation derived from the lexical meaning of the adjective and
$R_{2}$ a relation other than identity
Gastronomical book
$\lambda N \lambda x \exists e_{a} \exists e_{n} \cdot\left[\right.$ gastronomy $\left(e_{a}\right) \wedge$ about $\left.\left(e_{n}, e_{a}\right) \wedge N(\lambda S . S)\left(e_{n}\right)(x)\right](\lambda \operatorname{Pol} \lambda \operatorname{ex} x \cdot[\operatorname{Pol}(\operatorname{book}(e)) \wedge e=x])$
$\equiv \lambda x \exists e_{a} \exists e_{n} \cdot\left[\right.$ gastronomy $\left(e_{a}\right) \wedge$ about $\left.\left.\left(e_{n}, e_{a}\right) \wedge \operatorname{book}\left(e_{n}\right) \wedge e_{n}=x\right]\right)$

Figure 3: Semantics of Subsective Adjectives

$R$ relation mentioned in the general schema for adjective semantic representation.

Thus, the meaning of the adjectival phrase containing an adjective of measure, e.g. big mouse will be represented as:

$$
\begin{aligned}
& \lambda N \lambda x \exists e_{a} \exists e_{n} \cdot\left[\operatorname{size}\left(e_{a}\right) \wedge \text { highFor }\left(e_{a}, C\right)\right. \\
& \left.\wedge h a s\left(e_{n}, e_{a}\right) \wedge N(\lambda S . S)\left(e_{n}\right)(x)\right] \\
& (\lambda P o l \lambda e \lambda x \cdot[\operatorname{mouse}(e) \wedge e=x]) \\
& \equiv \lambda x \exists e_{a} \exists e_{n} \cdot\left[\operatorname{size}\left(e_{a}\right) \wedge \operatorname{highFor}\left(e_{a}, C\right)\right. \\
& \left.\left.\wedge h a s\left(e_{n}, e_{a}\right) \wedge \operatorname{mouse}\left(e_{n}\right) \wedge e_{n}=x\right]\right)
\end{aligned}
$$

where $C$ is a contextually given parameter which determine the scale size is measured against. In this case, $C$ would be, e.g. "mouse" so that the formula above can be glossed as $x$ is a mouse with a size $e_{a}$ which is high for a mouse. In particular, Daisy is a big mouse entails that Daisy is a mouse and that Daisy is big for a mouse, but not that Daisy is big.

\subsubsection{Privative adjectives}

As seen above, privative adjectives entail that the entity described by the NP is not N, e.g. a fake gun is not a gun. For such adjectives, it is the entity introduced by the adjective that is being quantified over, hence $e_{a}$ is identified with $x$ (cf. Figure 4). Fur- ther, the $\mathrm{N}$ property is either denied or subject to a modality (former, potential). As shown in Figure 4, this is accounted for by providing the appropriate relation $R$ (e.g. $R_{2}$ being the relation time introduced by former or $R_{1}$ being the identity relation $\mathrm{x}=e_{a}$ introduced by fake).

This representation presupposes that each sentence in which such modality adjectives do not occur has a default value for time and/or modality. Thus, for instance that

(7) John is a former president. $\forall \neq$ John is the president.

(8) John is a possible president. $\not \models$ John is the president.

can only be accounted for if the base forms are assigned the following default representations:

$$
\begin{aligned}
\text { (7) } \exists e_{a} \exists x & {\left[\operatorname{president}(x) \wedge \operatorname{time}\left(x, e_{a}\right)\right.} \\
\text { (8) } \exists e_{a} \exists x & \left.\wedge \text { present }\left(e_{a}\right)\right] \\
& \wedge \operatorname{president}(x) \wedge \bmod \left(x, e_{a}\right) \\
&
\end{aligned}
$$

\subsubsection{Plain non-subsective adjectives}

Finally, plain non-subsective adjectives fail to make any prediction about the existence of an in- 


\begin{tabular}{|l|}
\hline Privative Adjectives (e.g., fake,potential,former,future) \\
(e.g. fake, fictitious) \\
$\lambda N \lambda x \exists e_{a} \exists e_{n} \cdot\left[A\left(e_{a}\right) \wedge x=e_{a} \wedge N(\lambda S . \neg S)\left(e_{n}\right)(x)\right]$ OR \\
$\lambda N \lambda x \exists e_{a} \exists e_{n} \cdot\left[A^{\prime}\left(e_{a}\right) \wedge\right.$ mod/time $\left.\left(e_{a}, e_{n}\right) \wedge N(\lambda S . S)\left(e_{n}\right)(x)\right]$ \\
with $R_{2}$ being the relation mod/time specifying the modality or the time indicated by the adjective \\
Fake gun \\
$\lambda N \lambda x \exists e_{a} \exists e_{n} \cdot\left[\right.$ fake $\left.\left(e_{a}\right) \wedge x=e_{a} \wedge N(\lambda S . \neg S)\left(e_{n}\right)(x)\right](\lambda \operatorname{Pol} \lambda e \lambda x \cdot[\operatorname{Pol}($ gun $(e)) \wedge e=x])$ \\
$\equiv \lambda x \exists e_{a} \exists e_{n} \cdot\left[\right.$ fake $\left(e_{a}\right) \wedge x=e_{a} \wedge \neg$ gun $\left.\left.\left(e_{n}\right) \wedge e_{n}=x\right]\right)$ \\
Former president \\
$\lambda N \lambda x \exists e_{a} \exists e_{n} \cdot\left[\right.$ former $\left.\left(e_{a}\right) \wedge \operatorname{time}\left(e_{n}, e_{a}\right) \wedge N(\lambda S . S)\left(e_{n}\right)(x)\right]$ \\
$(\lambda P o l \lambda e \lambda x .[\operatorname{Pol}(\operatorname{president}(e)) \wedge e=x])$ \\
$\equiv \lambda x \exists e_{a} \exists e_{n}\left[\right.$ former $\left(e_{a}\right) \wedge$ time $\left(x, e_{a}\right) \wedge$ president $\left.\left(e_{n}\right) \wedge x=e_{n}\right]$ \\
\hline
\end{tabular}

Figure 4: Semantics of Privative Adjectives

dividual having the $\mathrm{N}$ property. Thus for instance, if John is an alleged murderer, there might or might not exist a murderer.

To account for this fact, we follow Hobbs' approach in distinguishing between existence in the universe of discourse and existence in the real world. Thus, the logical existential connective $\exists$ is used to denote existence in the discourse world while the special predicate Exists is used to denote existence in the real world. We assume further a theory that permits determining when an individual exists in the universe of discourse and when it exists in the real world.

Given these caveats, the semantics of plain nonsubsective adjectives is as indicated in Figure 5 and simply specifies that the alleged murderer is an individual $x$ which exists in the universe of discourse (but not necessarily in the real world) and which is alleged to be a murderer. Moreover, as stated in (Hobbs, 1985), we assume that the alleged predicate is existentially opaque in its second argument. That is, an alleged predication does not imply the existence in the real world of its second argument.

\section{Implementation}

The semantics of adjectives presented in this paper was tested using (Blackburn and Bos, 2005) computational semantics framework.

First, based on the classification of 300 English adjectives presented in (Amoia and Gardent, 2006), which identifies 17 different adjectival subclasses for the four main classes proposed by (Kamp, 1975; Kamp and Partee, 1995), we have built a test suite of about 150 examples in the following way. We have chosen for each class a representant adjective and written for it the set of sentence pairs $(\mathrm{H} / \mathrm{T})$ illustrating the inference patterns displayed by the class the adjective belongs to. In particular, we have built examples which test:

1. whether the adjective partecipates in both predicative and attributive constructions, so that the resulting sentences $(\mathrm{H}$ and $\mathrm{T})$ are paraphrastic,

2. whether the two sentences contain adjectives which are synonyms,

3. what kind of antonymic relation links the given adjective with its antonym,

4. which of the three inference patterns described in (Kamp and Partee, 1995) holds for the given adjective,

5. hyperonymy,

6. derivational morphology.

For instance, the test suite contains for an adjective such as fake, belonging to a subclass of the privative adjectives, the $\mathrm{H} / \mathrm{T}$ pairs in (9).

(9) a. H:This is a fake gun / T:This gun is fake 
Plain non subsective Adjectives (e.g., alleged)

$\lambda N \lambda x \exists e_{a} \exists e_{n} \cdot\left[A^{\prime}\left(e_{a}, e_{n}\right) \wedge x=e_{a} \wedge N(\lambda S . S)\left(e_{n}\right)\left(e_{n}\right)\right]$

with $R_{1}$ being the identity relation between $x$ and $e_{a}$ and $R_{2}$ being the relation

introduced by the adjective $A^{\prime}\left(e_{a}, e_{n}\right)$

Alleged murderer

$\lambda N \lambda x \exists e_{a} \exists e_{n} .\left[\right.$ alleged $\left.\left(e_{a}, e_{n}\right) \wedge x=e_{a} \wedge N(\lambda S . S)\left(e_{n}\right)\left(e_{n}\right)\right](\lambda \operatorname{Pol} \lambda e \lambda x .[\operatorname{Pol}($ murderer $(e)) \wedge e=x])$ $\equiv \lambda x \exists e_{a} \exists e_{n} .\left[\right.$ alleged $\left.\left.\left(e_{a}, e_{n}\right) \wedge x=e_{a} \wedge \operatorname{murderer}\left(e_{n}\right) \wedge e_{n}=e_{n}\right]\right)$

Figure 5: Semantics of plain non-subsective Adjectives

b. H:This is a fake gun / T:This is a false gun

c. H:This is a fake gun / T:This gun is not genuine

d. H:This is not a fake gun $=$ This gun is real

e. H:This is a fake gun / T:This is a gun

f. H:This is a fake gun / T:This is not a gun

g. H:This is a fake gun / T:This is fake

h. H:This is a fake gun / T:This is a fake weapon

i. H:This is a fake gun / T:This gun is a counterfeit

Second, a grammar fragment was implemented which integrates the semantics of nouns and adjectives presented here. This grammar fragment was then used together with the appropriate lexicon to automatically associate with each sentence of the test suite a representation of its meaning.

Third, lexical Knowledge pertaining to each class of adjectives is captured through a set of axioms describing the specific lexical relationships adjectives are involved in.

Synonymy is captured introducing equality axioms which describe the equivalence of the two properties expressed by the two adjectives $A d j_{1}$ and $A d j_{2}$ asserting:

$$
\forall e\left[\operatorname{Adj}_{1}(e) \leftrightarrow \operatorname{Adj}_{2}(e)\right]
$$

Hyponymy (for example big/giant vs. small/minuscule) is captured by introducing the axioms such as:

$$
\forall e\left[\operatorname{Adj}_{1}(e) \rightarrow \operatorname{Adj}_{2}(e)\right]
$$

Antonymy is captured by introducing different axioms depending on the type of opposition relation in which the adjectives are involved, i.e. binary, contrary or multiple opposition. The axiom below for example introduces a binary antonymic relation:

$$
\forall e\left[\operatorname{Adj}_{1}(e) \leftrightarrow \neg \operatorname{Adj}_{2}(e)\right]
$$

Fourth, entailment $(\mathrm{H} \mid=\mathrm{T})$ was checked for each sentence pair using the first order theorem provers available in the system and the results compared with the expected result. A first evaluation shows that the methodology proposed yields the expected results: we could correctly predict all the inferential patterns presented above from 1 to 5 (136 pairs, $89 \%$ ). The results for other patterns, describing morphoderivational relations of adjectives, depend on the amount of information implemented in the grammar which for the moment is very limited.

\section{Perspectives and Comparison with related works}

The approach presented here lays the basis for a computational treatment of adjectival inference in that it provides a fine grained characterisation of the various types of inferential patterns licenced by adjectives.

In future work, we believe three main points are worth investigating.

First, previous work (Amoia and Gardent, 2006) has shown that the classification presented here can be further detailed and even finer-grained classes identified thereby permitting the creation of syntactically and semantically homogeneous adjectival 
classes. The advantages of identifying such homogeneous classes has been well demonstrated for verbs. It permits structuring the lexicon and facilitates development and maintenance. Based on the idea that syntax (and in particular, so-called syntactic alternations) helps define such classes, we are currently investigating in how far adjectival syntax helps further refine adjectival classes.

Second, the proposed classification need to be applied and combined with ontological and lexical semantic information. That is, each adjective should be classified wrt the 4 types of model theoretic semantics described here and related to such a lexical semantics ontology as e.g., WordNet, the MikroKosmos ontology of the SIMPLE lexicon.

Thus (Raskin and Nirenburg, 1995) describe the methodology used to encode adjectival entries in the lexicon of the MikroKosmos semantic analyser. The MikroKosmos lexicon contains 6,000 entries for English and 1,500 entries for Spanish adjectives. Adjectives are organised in an ontology which distinguishes between the following three main adjectival classes: (i) Scalar Adjectives, which are represented as property-value pairs, (ii) Denominal Adjectives, (e.g. atomic, civil, gastronomical) represented as nouns and (iii) Deverbal Adjectives, (e.g. eager, abusive, readable) is related to the meaning of the verb they are derived to.

The classification of adjectives proposed in SIMPLE (SIMPLE, 2000) is also ontology-based. A lexical entry for an adjective is characterised by a set of semantic and syntactic information. Semantic information describes: (i) the hierarchy of ontological properties expressed by the particular adjective, for example the adjective expresses the property of COLOUR and this is a physical property; (ii) whether the adjective is intersective or subsective; (iii) whether the adjective has a persistent duration (i.e. is stable) or not. Moreover, syntactic information describes adjectival features such as (i) predicative/attributive usage, and (ii) gradability.

SIMPLE has actually added semantic information to approximately 3,500 lexical entries (about 10,000 senses) for each of the 12 European languages considered in the project.

It would be interesting to see whether any of these resources can be used to create an adjective lexicon rich enough to support both syntactic processing and semantic inference.

Finally, a third point of interest concerns the integration of the compositional semantics proposed here for adjectives into a robust semantic processing system. We plan to integrate this semantics into the CCG2Sem semantic parsing system (Bos, 2005) and to investigate in how far, this would help deal with entailment recognition.

\section{References}

Marilisa Amoia and Claire Gardent. 2006. Adjective based inference. In Proceedings of KRAQ'06 (Knowledge and Reasoning for Answering Questions), Trento, Italy.

Patrick Blackburn and Johan Bos. 2005. Representation and Inference for Natiral Language. A first Course in Computational Semantics. CSLI Studies in Computational Linguistics.

Johan Bos. 2005. Towards wide-coverage semantic interpretation. In In Proceedings of the Sixth International Workshop on Computational Semantics IWCS6 , pages 42-53.

G. Chierchia and S. Mc Connell-Ginet. 1990. Meaning and Grammar: An Introduction to Semantics. The MIT Press, Cambridge, MA.

Jerry R. Hobbs. 1985. Ontological promiscuity. In Proceedings of the 23rd Annual Meeting of the Association for Computational Linguistics, pages 61-69, Chicago, Illinois, July.

Hans Kamp and Barbara Partee. 1995. Prototype theory and compositionality. Cognition, (57):129-191.

Hans Kamp. 1975. Two theories about adjectives. In Edward L. Keenan (ed.), Formal Semantics of Natural Language, pages 123-155. Cambridge University Press.

V. Raskin and S. Nirenburg. 1995. Lexical Semantics of Adjectives, a micro-theory of adjectival meaning. MCCS Report.

Specification Group SIMPLE. 2000. Specification simple work package 2. Linguistic specifications deliverable d2.1. 